\title{
Sistema para ejecutar políticas sobre infraestructuras de Tecnologías de la Información
}

\author{
IT policies execution system
}

\author{
Mónica Peña Casanova ${ }^{1 *} \quad$ Caridad Anías Calderón ${ }^{2}$
}

Recibido 16 de febrero de 2018, aceptado 20 de agosto de 2018.

Received: February 16, 2018 Accepted: August 20, 2018

\begin{abstract}
RESUMEN
Una vía eficaz para lograr el alineamiento entre las infraestructuras de las tecnologías de la información y los objetivos de la organización es la ejecución de políticas, que se definen al más alto nivel ejecutivo. Este proceso no resulta sencillo por cuanto se deben traducir dichas políticas a reglas que puedan ser interpretadas por los recursos gestionados y debe garantizarse una retroalimentación efectiva para la evaluación de su cumplimiento. El presente trabajo plantea un sistema que incluye un procedimiento para la ejecución de las políticas de la organización sobre infraestructuras de las tecnologías de la información y una arquitectura de gestión de redes basada en políticas para su automatización. A través de la integración de la gestión basada en políticas y los acuerdos de nivel de servicio, dicho sistema facilita la implementación de las políticas de la organización, que son automatizables sobre las infraestructuras TI y la evaluación del cumplimiento de éstas.
\end{abstract}

Palabras clave: Gestión de TI, PBNM, acuerdos de nivel de servicio, SDN híbrida.

\begin{abstract}
The execution of high level design policies, over IT infrastructure is an effective method to enhance ITbusiness strategic alignment. This is not an easy process because these policies must be translated into rules that can be interpreted by the managed resources and, in addition, an effective feedback of the impact of their fulfillment must be guaranteed. In this paper we present a system that includes a procedure for executing the organization's policies on IT infrastructures and, as part of it, an architecture based on PBNM for the automation of its execution. Through the integration of policy-based management and service level agreements, this system addresses the following: the implementation of the organization's policies, which are automated on IT infrastructures and the evaluation of compliance with themselves.
\end{abstract}

Keywords: IT management, PBNM, SLA, hybrid SDN.

\section{INTRODUCCIÓN}

Las infraestructuras TI se han transformado en un activo estratégico para las organizaciones por cuanto les permiten la mejora de los procesos de negocio, la reducción de los costos y la creación de productos y servicios innovadores, garantizando el cumplimiento de los requerimientos regulatorios siempre en aumento [1-2]. Dichas infraestructuras abarcan una serie de recursos que incluyen: el hardware y el software

\footnotetext{
1 Universidad de las Ciencias Informáticas. La Habana, Cuba. E-mail: monica@uci.cu

2 Universidad Tecnológica de La Habana "José Antonio Echeverría". La Habana, Cuba. E-mail: cacha@tesla.cujae.edu.cu

* Autor de correspondencia: monica@uci.cu
} 
de las computadoras, las tecnologías de redes, las aplicaciones para el procesamiento de datos, los servicios telemáticos, los datos, así como los recursos humanos de TI con sus habilidades.

Para lograr los beneficios antes mencionado, es necesario garantizar la alineación de las infraestructuras TI que se emplean, con los objetivos de la organización. Una vía para hacerlo es la definición de políticas TI. Una política TI es una combinación de reglas que definen los criterios para el acceso y utilización de recursos que forman parte de las infraestructuras TI, que debe servir de guía a la hora de establecer y priorizar objetivos y oportunidades. Para el establecimiento de políticas es preciso contar con un sistema organizacional en el que estén claramente delimitados los niveles de autoridad y responsabilidad [3]. Estas políticas pasan por un proceso de estratificación, que parte de su definición al más alto nivel, la traducción de estas a un lenguaje apto para ser interpretado por los sistemas informáticos, expresadas en reglas (evento condición acción), que deben evaluar las condiciones para la aplicación; y ejecución sobre los recursos gestionados de las acciones definidas [3]. Para la automatización de este objetivo, una alternativa es la gestión de red basada en políticas (PBNM por las siglas en inglés de Policy Based Network Management) la cual permite controlar y coordinar, de manera dinámica, los elementos gestionados tomando decisiones de forma automática a través de reglas y de peticiones de usuarios o de servicios. Sin embargo, la interpretación de dichas reglas por los recursos gestionados de acuerdo a sus características, no resulta un proceso sencillo, por cuanto implica la aceptación por el equipamiento y servicios heterogéneos existentes y la solución de conflictos entre ellas [4].

De lo anterior se plantea la necesidad de diseñar un sistema para la ejecución de las políticas de la organización sobre las infraestructuras TI, que permita el aseguramiento tecnológico de los procesos claves de la organización y sus estrategias. Dicho sistema, a nivel tecnológico, debe permitir, realizar una gestión integrada basada en políticas, la traducción de las reglas a las características de los recursos gestionados y la solución de conflictos entre estas, mediante una adecuada gestión de los Acuerdos de Nivel de Servicio y la evaluación de un conjunto de mediciones que forman parte de él [5-6].
En el presente artículo se desarrolla un sistema consistente en un procedimiento y una arquitectura para la ejecución de políticas de la organización sobre las infraestructuras TI, que permite lograr la alineación de estas a los objetivos de cada organización.

\section{MATERIALES Y MÉTODOS}

Durante la realización de la presente investigación se utilizaron los métodos: analítico - sintético: durante la descomposición del problema de investigación en elementos, la profundización en el estudio de cada uno y la sintetización en la solución propuesta; el histórico-lógico y dialéctico, utilizado durante el estudio crítico de la evolución de los diferentes enfoques relativos a la gestión de redes y servicios telemáticos, para emplearlos como punto de referencia y comparar los resultados alcanzados y la modelación para el diseño del sistema consistente en un procedimiento y una arquitectura.

Para la formulación de políticas de TI a partir de las políticas de una organización, debe realizarse de un diagnóstico, a través del cual se precise: cuáles son los objetivos y prioridades, cuál es el modelo de negocios, qué alianzas o relaciones de cooperación va a establecer la organización, cuáles son los flujos de información y materiales tanto internos como externos que forman parte de su funcionamiento, cuáles son las fuentes de recursos humanos, materiales y financieros de la entidad y, conocer las métricas para evaluar el cumplimiento de los objetivos. Adicionalmente, deben tomarse en cuenta requerimientos legales y regulaciones asociados a las TI, así como requerimientos técnicos y contractuales entre proveedores y clientes de TI [7].

En la definición de la política se debe incluir: objetivos, prioridades, alcance, descripción, responsables, indicadores, excepciones, sanciones, así como los mecanismos de divulgación. Las políticas pueden ser de configuración, de uso, de seguridad, de instalación, de respuesta ante eventos, entre otras. Para lograr una representación holística del entorno gestionado, las políticas deben ser estratificadas de manera tal que en cada capa se represente un dominio de gestión, lo cual facilitará su implementación y evaluación. Para lograr una representación holística del entorno gestionado, las políticas deben ser estratificadas de manera tal que 
en cada capa se represente un dominio de gestión, lo cual facilitará su implementación y la evaluación del impacto. Para ello, Stassner [8] proponen el Modelo de Estratificación de Políticas que garantiza el seguimiento de dichas políticas desde los niveles estratégicos hasta las instancias en las que estas se ejecutan [3], [9]. Esto se muestra en la Figura 1. El modelo de estratificación de políticas propuesto de Strassner forma como un árbol recursivo de objetos de políticas en el que cada política queda enlazada con las políticas correspondientes de niveles inferiores, siendo posible que una política de nivel inferior este enlazada a varias de niveles superiores.

Fuente: [8].

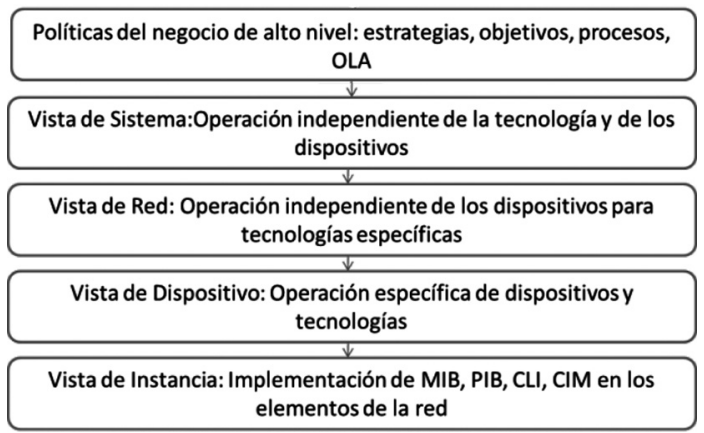

Figura 1. Estratificación de políticas.

De acuerdo a la capacidad de automatizarse, las políticas de TI pueden clasificarse a dos grupos, las que pueden automatizarse total o parcialmente y las que no. Las no automatizables deberán ejecutarse a través de medidas organizativas que no se detallarán como parte de este artículo. Una regla de política automatizable contiene metadatos que definen el comportamiento y función de la regla, la cláusula de condición y la cláusula de acción [10].

La mayor parte de las políticas automatizables se convierten en tareas de operación en las infraestructuras TI que se llevan a cabo, utilizando una ventana de tiempo en la cual pueden realizarse cambios sin afectar el negocio. Las soluciones PBNM permiten realizar gran cantidad de cambios en esa ventana de tiempo, facilitando la automatización de tareas complejas y repetitivas [11].

Las soluciones PBNM utilizan la arquitectura definida por el Grupo de Trabajo de Internet (IETF por las siglas en inglés Internet Engineering Task Force)
[13], que se muestra en la Figura 2. En la misma se aprecian diferentes tipos de entidades: herramienta de gestión de políticas, contenedor o repositorio de políticas, punto de decisión de políticas o servidor de políticas (PDP por las siglas en inglés Policy Decision Point) y uno o varios puntos de ejecución de políticas (PEP por las siglas en inglés Policy Enforcement Point).

El agente PDP revisa las políticas almacenadas en el contenedor de políticas y efectúa un proceso de toma de decisiones. El PDP envía las decisiones tomadas, que son independientes de las características de los dispositivos a los PEP asociados, que se encargan de traducirlas en operaciones o comandos específicos que puedan ser interpretados por la tecnología concreta de los agentes que actúan en los recursos gestionados por dichos PEPs.

En la herramienta de gestión de políticas, estas se modelan de acuerdo al ciclo de vida que establece la RFC 3198. Este ciclo adolece de la detección y solución de conflictos entre políticas, lo cual resuelve el sistema que se presenta en el artículo [4], [14].

No obstante, la automatización de la ejecución de políticas, por sí misma, no garantiza la alineación de las infraestructuras TI con los objetivos de las organizaciones, es preciso, además llevar a cabo un

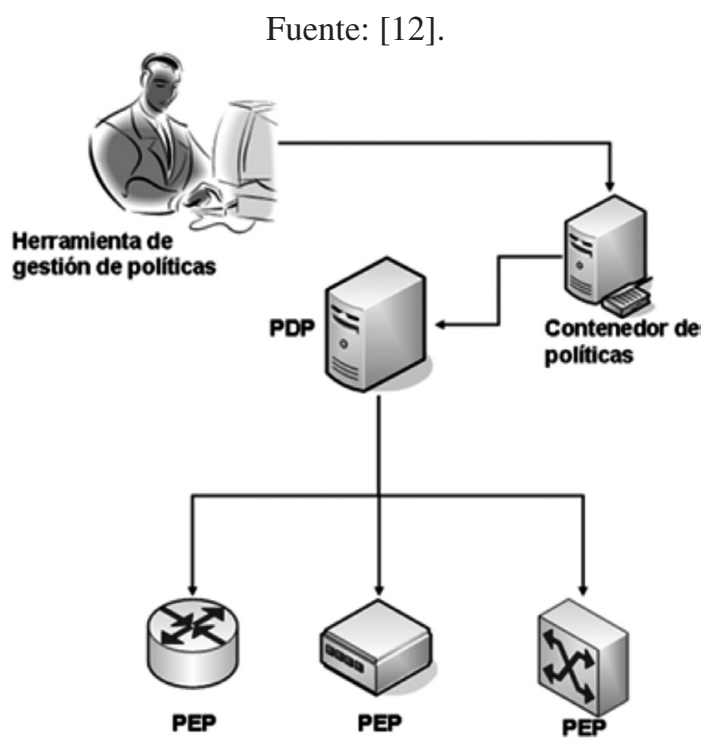

Figura 2. Arquitectura PBNM propuesta por el IETF. 
grupo de actividades que permita su cohesión con los objetivos del negocio mediante la vinculación en los departamentos de TI y los departamentos funcionales de la organización [3, 7]. En este contexto se propone la adopción de Acuerdos de Nivel de Servicio (SLA del inglés Service Level Agreement). Los SLA crean un lenguaje común y comprensible entre el área TI y el resto de las áreas de procesos de la organización, describiendo los servicios que proveerá la primera, las responsabilidades de cada una de las partes, permitiendo la precisión de indicadores en las instancias de los servicios que reciben estas últimas y la comparación de los mismos con los niveles de calidad esperados. En tanto, los departamentos de TI cuentan con métricas para demostrar el cumplimiento de su misión y herramientas para dar seguimiento al funcionamiento de los servicios que gestionan [15].

La gestión de SLA, considera la integración de cinco fases: desarrollo o diseño del servicio, negociación, implementación, ejecución y aseguramiento, como se aprecia en la Figura 3 [12].

Para facilitar la adopción de SLA en las organizaciones, existen diferentes marcos referenciales como los Objetivos de Control para las Tecnologías de la Información (COBIT por sus siglas en inglés), la Biblioteca de Infraestructura de Tecnologías de Información (ITIL por sus siglas en inglés)y el Mapa mejorado de Operación para las Telecomunicaciones (eTOM por sus siglas en inglés), que permiten medir de manera individual aspectos relevantes de las TI y que pueden contribuir al logro de los objetivos de negocio dentro de la organización. En la presente investigación se acoge la propuesta de ITIL en su versión 3 por ser la más genérica y la que mejor se enfoca en la mejora continua de los servicios desplegados [17-20].

Realizar de manera sistémica la conjugación de políticas definidas al más alto nivel, que responden a los objetivos de las organizaciones, con cada uno de los componentes que forman parte de las infraestructuras TI, automatizarlas a través de la PBNM y emitir informes sobre el cumplimiento de Acuerdos de Nivel de Servicio que se negocian entre las áreas de TI y el resto de las áreas funcionales de las organizaciones, no resulta sencillo. Es necesario definir variables de control de cada una de las etapas y que permitan evaluar la factibilidad de realizar la automatización de las políticas de las organizaciones, la solución de conflictos entre ellas y su perfeccionamiento. Para lograr esto, se plantea un procedimiento para la ejecución de las políticas de la organización sobre infraestructuras TI y, como parte del mismo, una arquitectura basada en PBNM para la ejecución de las políticas.

\section{RESULTADOS}

Para la concepción del sistema, se tuvieron en cuenta principios y premisas que garantizan la factibilidad del mismo. Entre los principios considerados, a cumplir por el sistema, se pueden destacar los siguientes:

- Alta estandarización y neutralidad tecnológica a partir del cumplimiento de marcos de referencia.

- Flexibilidad de su aplicación, para adaptarse a diferentes escenarios TI.

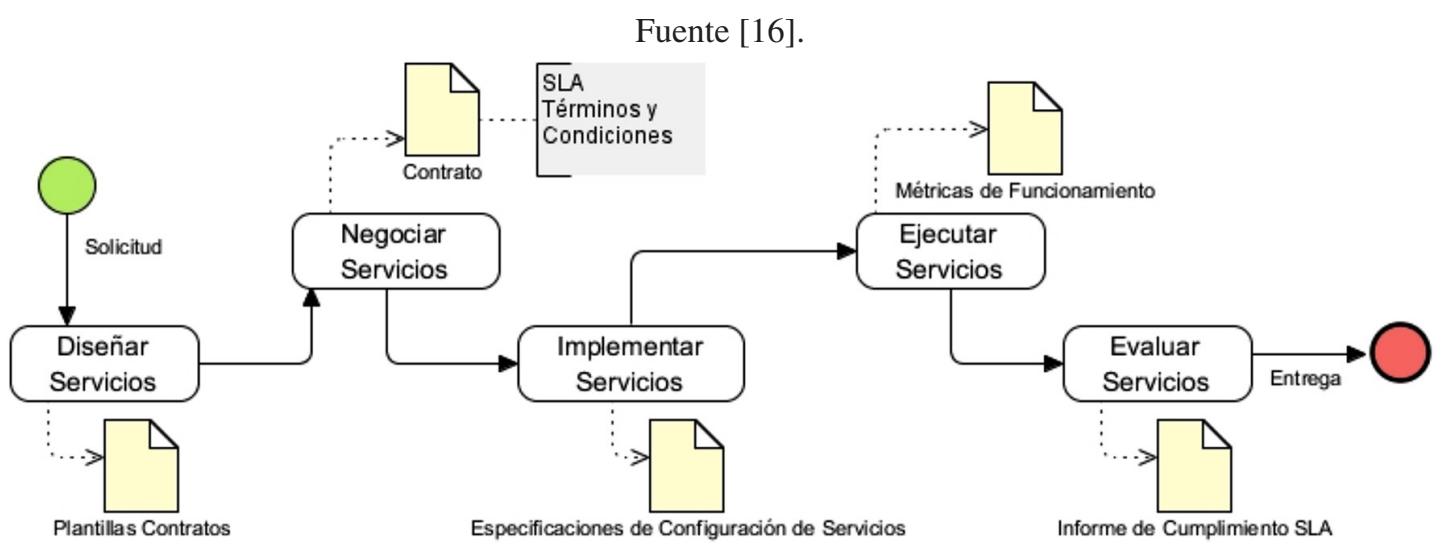

Figura 3. Fases para el establecimiento de Acuerdos de Nivel de Servicio. 


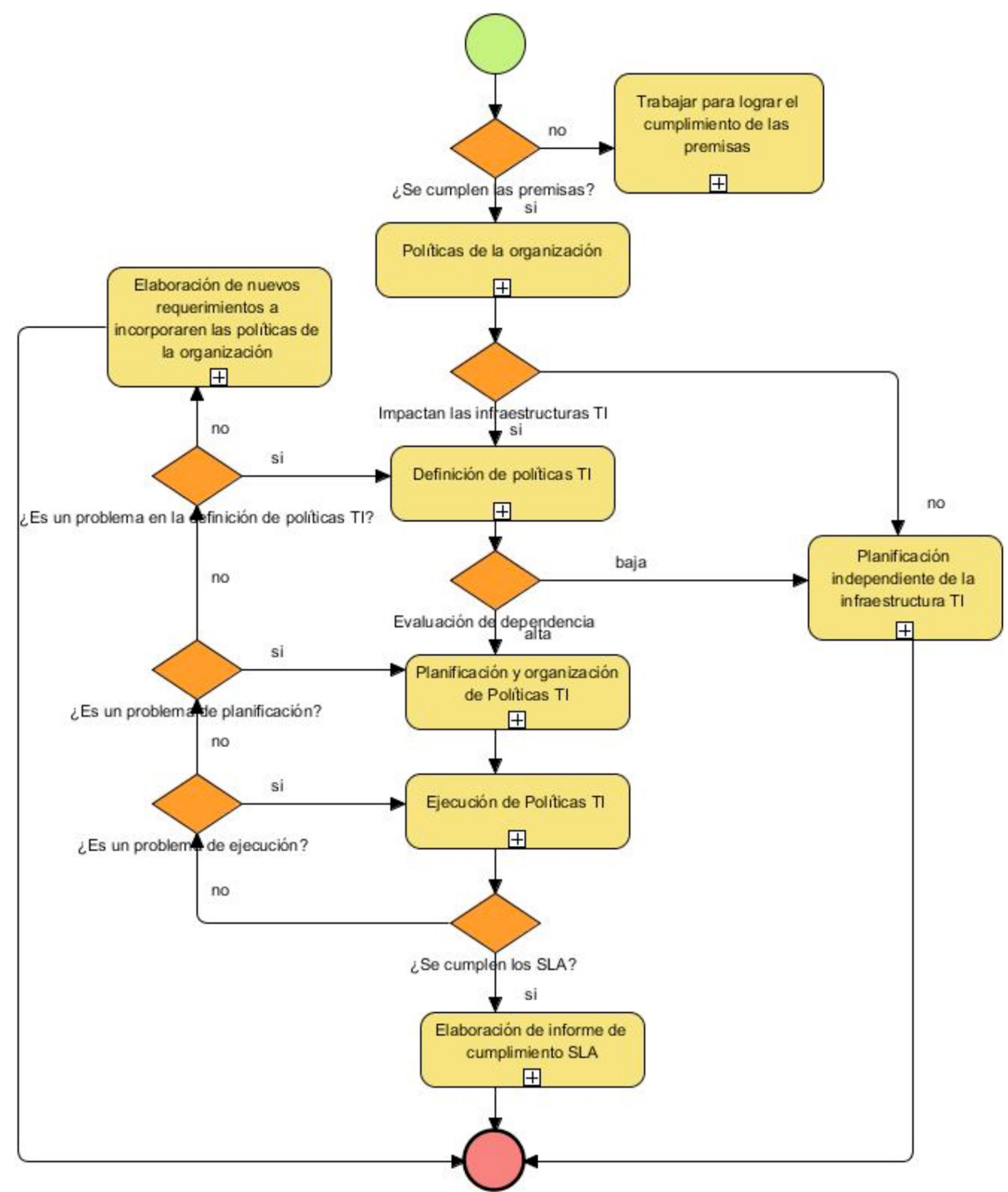

Figura 4. Procedimiento general para aplicación de políticas de la organización sobre la infraestructura TI.

- Pertinencia expresada en el alineamiento de las TI a las políticas de la organización a partir del diseño de Acuerdos de Nivel de Servicio.

- Mejora continua y adaptación a las necesidades cambiantes de la organización a partir de la evaluación de políticas y la solución de conflictos entre éstas.

Se consideraron un conjunto de premisas para la implementación del sistema, las cuales permiten la ejecución de las políticas de la organización sobre las infraestructuras TI. En primer lugar, es preciso que el personal que opera las TI debe estar familiarizado con los objetivos de la organización y debe ser altamente calificado para la gestión de la infraestructura TI. Esto se expresa garantizando que más del 70\% del personal de TI este capacitado en las tecnologías desplegadas. En segundo lugar, la organización debe intencionar la adopción de estándares. El 100\% de los procesos asociados a las cinco áreas de procesos definidas por COBIT 5, determinados, con un nivel de capacidad 1: Proceso ejecutado, para COBIT 5 y la ISO/IEC 15504 [14]. Es también necesario que la infraestructura TI sea 
gestionable, para lo cual, es necesario que más del $80 \%$ del equipamiento sea gestionable y el $100 \%$ del equipamiento asociado a los servicios críticos deben estar definidos como parte de los requerimientos de servicios negociados en los SLA que se observan en la Figura 3, tiene que ser gestionable. Además, la infraestructura TI deben contar con capacidades suficientes para implementar la automatización de las políticas, o sea su ejecución en las infraestructuras TI, lo cual se garantiza mediante la disponibilidad de hardware para despliegue de soluciones de gestión y uso de ancho de banda. Estos valores estarán en función de las métricas de desempeño definidas por TL 9000 para el sector de la industria al que pertenece la organización [17-18]. Finalmente, deben estar formalizadas a través de procedimientos las tareas de gestión de las infraestructuras TI, lo que implica que el $80 \%$ de las tareas de gestión deben estar procedimentadas y el $100 \%$ de las asociadas a la continuidad del negocio.

El personal que opera las TI debe ser capaz de proponer a los directivos de la organización un amplio portafolio de servicios alineado con las necesidades de ésta y, a la vez deben tener sistematizadas las tareas de gestión a ejecutar y contar con un amplio dominio de las tecnologías que forman parte de la infraestructura que gestionan.

La adopción de estándares en la organización la dotará de capacidades como la interoperabilidad, facilitará la adopción de buenas prácticas que podrán sistematizarse y permitirá la reducción de la complejidad en infraestructuras heterogéneas.

Que la infraestructura TI sea gestionable, significa que debe existir una base de datos de gestión de configuración y que se mida el desempeño de los componentes de la infraestructura.

Para ejecutar políticas de manera automatizada es necesario destinar una parte de la infraestructura TI a este fin, de ahí que sea necesario determinar si ésta cuenta con capacidades suficientes para ello o se puede invertir con este propósito.

Para evaluar la factibilidad de ejecutar el procedimiento que permite ejecutar políticas de la organización en infraestructuras TI, debe verificarse el cumplimiento de las premisas antes enunciadas, mediante la verificación de las mediciones que las componen.
Como se observa en la Figura 4, el procedimiento para la ejecución de las políticas de la organización sobre las infraestructuras TI, consta tareas, las cuales tienen medidas asociadas que se instanciarán a cada organización.

Como se observa en la Figura 4, una vez definidas las políticas de la organización se evalúan cuáles impactan las infraestructuras TI y cuáles no. Como se observa en la Tabla 2, impactan las infraestructuras TI aquellas políticas que implican mantener información de calidad para apoyar las decisiones del negocio, generar valor organizacional mediante los servicios habilitados por las TI, o sea, obtener metas estratégicas y mejoras al negocio mediante el uso eficaz e innovador de la TI, lograr excelencia operativa mediante la aplicación eficiente y fiable de la tecnología, mantener el riesgo relacionado con TI a niveles aceptables y optimizar el costo de la tecnología y los servicios de TI. Es recomendable evaluar la dependencia (D) de la organización con las TI, ver (1), lo cual está en función del porciento de políticas de la organización que impactan en las infraestructuras TI $\left(\mathrm{Po}_{\mathrm{TI}} / \mathrm{Po}\right)$ y de la prioridad de estas políticas $(p)$ que está relacionada con el porcentaje de programas alineados con los requisitos / prioridades de negocio corporativos, definido en el APO08 de COBIT 5: Procesos catalizadores [14]. Dicha prioridad está en función de la agilidad que aportarán las políticas de la organización que impactan en las infraestructuras TI en el cumplimiento de la misión de la organización y en la continuidad del negocio.

$$
D=p\left(\frac{P o_{T I}}{P o}\right) 100
$$

Siendo:

$P O_{\mathrm{TI}}:$ Políticas de TI.

Po: Políticas organizacionales.

$p$ : prioridad de las políticas.

A partir de las políticas de la organización que impactan en las infraestructuras TI, se realiza el diseño de las políticas TI. La semántica de qué cambiar, cuándo hacerlo y quién lo hará, puede representarse mediante reglas o procesos de negocio y debe ponerse en función de manejar la configuración de la infraestructura TI que se construye y despliega. Deben utilizarse dos mapeos, uno entre los objetivos 
Tabla 1. Medidas relacionadas con la ejecución del procedimiento.

\begin{tabular}{|c|c|}
\hline Tareas & Medidas Relacionadas \\
\hline $\begin{array}{l}\text { Evaluar políticas de la } \\
\text { organización que impacta } \\
\text { en las infraestructuras TI. }\end{array}$ & $\begin{array}{l}\text { \% de políticas de la organización soportadas por infraestructuras TI. } \\
\text { Información de procesos organizacionales captada, procesada o disponible a través } \\
\text { de un sistema informático. } \\
\text { Impacto de las TI en el negocio. }\end{array}$ \\
\hline $\begin{array}{l}\text { Diseñar políticas y } \\
\text { estrategias para las TI. }\end{array}$ & $\begin{array}{l}\% \text { de políticas TI mapeadas con políticas de la organización. } \\
\% \text { de tiempo que se utiliza en la ejecución de políticas en la infraestructura. } \\
\% \text { de tiempo que se utiliza en la corrección de errores. } \\
\% \text { de políticas TI automatizables. }\end{array}$ \\
\hline Evaluar dependencia. & $\begin{array}{l}D=p\left(P o_{\mathrm{TI}} / P o\right) 100 . \\
\mathrm{D}: \text { Dependencia de la organización con las TI. } \\
P o_{\mathrm{TI}}: \text { Políticas de TI. } \\
P o: \text { Políticas organizacionales. } \\
p: \text { prioridad de las políticas para la organización. }\end{array}$ \\
\hline
\end{tabular}

de la organización y las metas a alcanzar en las TI y otro explicando cómo se va a implementar las acciones para alcanzar dichas metas, de manera tal que puedan definirse reglas de negocio ajenas a los términos de red, que se instanciarán hasta establecer cómo se construyen y despliegan configuraciones en dispositivos y sistemas, para lo cual es necesario el uso detallado de términos técnicos.

\section{FASE PLANIFICACIÓN, ORGANIZACIÓN DE POLÍTICAS}

Esta fase tiene como objetivo, diseñar políticas de TI alineadas a las necesidades de la organización y establecer las condiciones que propicien el cumplimiento de dichas políticas y la satisfacción de los objetivos de la organización.

Tal y como muestra la Figura 5, el primer paso consiste en realizar un diagnóstico de la organización, evaluar el nivel de desarrollo de los catalizadores definidos por COBIT5. Los catalizadores no son más que recursos organizativos para el gobierno de las infraestructuras TI, entre ellos se destacan: los marcos de referencia, principios, estructuras, procesos y prácticas, asî como recursos corporativos como capacidades de servicios, personas e información. En este

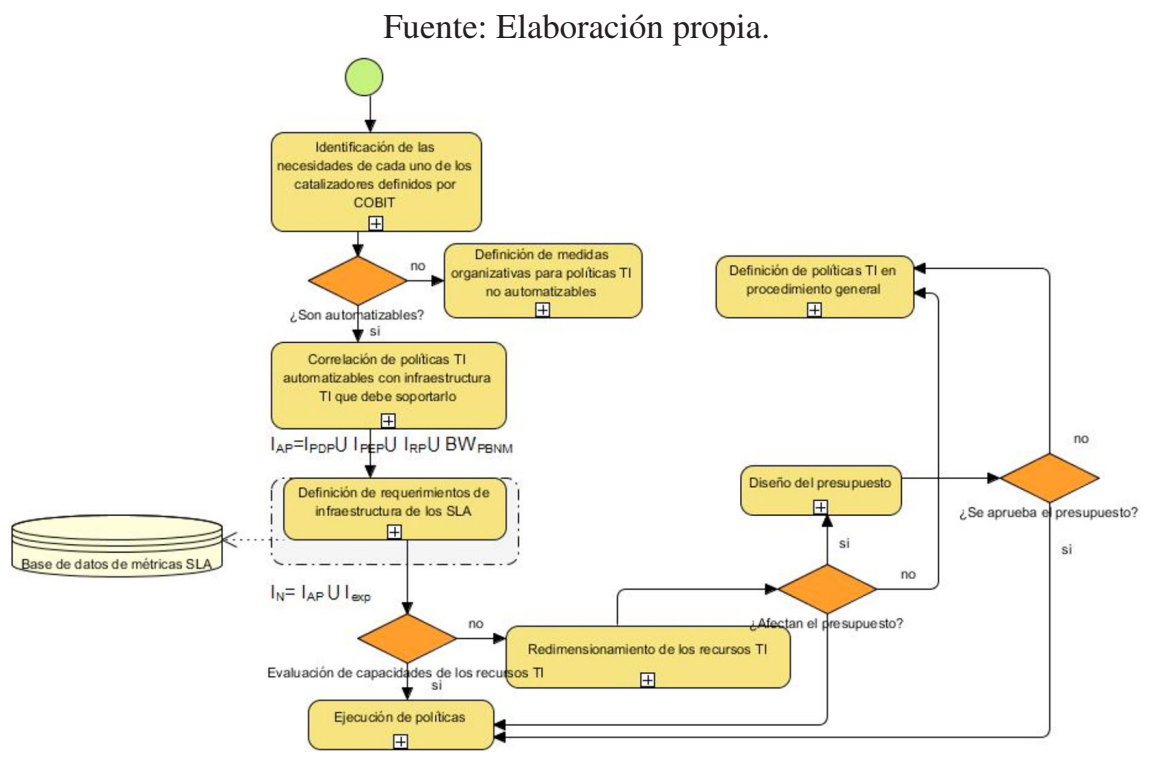

Figura 5. Planificación, Organización de Políticas. 
caso, como se observa en la Figura 5, resulta una buena práctica determinar las necesidades de cada uno de los catalizadores de la empresa teniendo en cuenta que están interconectados entre sí. Es decir, los procesos necesitan información, las estructuras organizacionales necesitan recursos humanos con habilidades y comportamientos, además, esta relación es bidireccional, puesto que los procesos entregan información y los recursos humanos con sus habilidades, mejoran la eficiencia de los procesos [19]. Por otra parte, para evaluar la factibilidad de ejecutar el procedimiento para la ejecución de las políticas de la organización sobre las infraestructuras TI, se recomienda calcular el tiempo medio de ejecución de las políticas en la infraestructura como la sumatoria del tiempo de las tareas asociadas a dicha ejecución entre el fondo de tiempo del personal T, según (2).

$$
F_{\alpha c}=\sum_{i=1}^{n} T_{e p}(i) \div T_{t}
$$

Donde:

$F_{\text {ac }}$ : es la factibilidad de ejecutar el procedimiento.

$T_{\mathrm{ep}}$ : es el tiempo medio de ejecución de políticas.

$T_{\mathrm{t}}$ : $\quad$ es el fondo de tiempo del personal de TI.

$N$ : es el total de tareas asociadas a la ejecución de políticas.

Es necesario evaluar la capacidad que posee la infraestructura para garantizar el cumplimiento de los objetivos de la organización. Para evaluar la infraestructura requerida para alcanzar los niveles de calidad de servicio deseados $\left(I_{\text {exp }}\right)$ dependen de la infraestructura necesaria para alcanzar los atributos de funcionamiento de la red $\left(I_{\mathrm{FN}}\right)$ y la infraestructura necesaria para conseguir el nivel de confianza que se planifica en la red $\left(I_{\mathrm{NC}}\right)$. Para determinar la infraestructura necesaria para tener un nivel de confianza en la red, debe modelarse la infraestructura subyacente capaces de afrontar deficiencias temporales o permanentes en el funcionamiento de los servicios para garantizar las transiciones alta/baja, falla/ reparación y reconfiguración, determinados por métricas como la disponibilidad, seguridad y confiabilidad de los servicios. Los atributos de funcionamiento de la red modelan el comportamiento de un sistema bajo el régimen de sin averías para derivar medidas de rendimiento relacionadas con tiempo de respuesta, rendimiento y utilización. Para el cálculo de $I_{\text {exp }}$ ver (3).

$$
I_{\text {exp }}=I_{N C} \cup I_{F N}
$$

Una vez obtenida la infraestructura necesaria para la prestación de servicios con los niveles de calidad diseñados, para automatizar la ejecución de políticas es necesario desplegar una infraestructura adicional $\left(I_{\mathrm{AP}}\right)$, esta infraestructura debe ser independiente de la que soporta los servicios, para garantizar la recuperación ante fallos y la respuesta ante eventos que deterioren las prestaciones en los servicios de ahí que la infraestructura necesaria $\left(I_{\mathrm{N}}\right)$ se obtiene de la unión de $I_{\mathrm{AP}}$ e $I_{\text {exp }}$, tal y como se muestra en (4).

$$
I_{N}=I_{A P} \cup I_{\exp }
$$

Donde:

$I_{\mathrm{N}}$ : es la infraestructura que se necesita.

$I_{\mathrm{AP}}$ : es la infraestructura necesaria para la automatización de políticas.

$I_{\text {exp }}$ : es la infraestructura en explotación de los servicios con nivel de calidad deseado.

Para determinar la infraestructura que se necesita para la automatización de políticas, es necesario conocer la cantidad de puntos de decisión de políticas que se necesitan y si existen, además de la demanda de procesamiento que estos tendrán, $\left(I_{\mathrm{PDP}}\right)$ en el caso de trabajar de manera autonómica, esto dependerá del mecanismo que se implemente para la toma de decisiones. También, debe determinarse la infraestructura que se requiere en el repositorio de políticas $\left(I_{\mathrm{RP}}\right)$ determinada por la capacidad de almacenamiento y la estructura de la base de datos que se utilizará para el almacenamiento de políticas, en esta medida, también impactará el modelo de información que se utilice para la representación de políticas, lo cual se abordará en el epígrafe que sigue. El despliegue de la arquitectura de gestión basada en políticas implica consumo de ancho de banda para la ejecución de políticas y para la verificación del funcionamiento de los elementos gestionados $\left(B W_{\mathrm{PBNM}}\right)$. Finalmente, es necesario determinar la infraestructura que se requiere en los puntos de ejecución de políticas $\left(I_{\mathrm{PEP}}\right)$ determinada por la capacidad de procesamiento o del hardware requerido para la instalación de los agentes en los elementos gestionados que permitirá la ejecución de políticas en éstos (5).

$$
I_{A P}=I_{P D P} \cup I_{P E P} \cup I_{R P} \cup B W_{P B N M}
$$


Como se observa en la Tabla 3, deben evaluarse los recursos humanos y materiales y determinar si es preciso tomar medidas organizativas o tecnológicas para lograr el alineamiento con las necesidades de la organización. A partir de la evaluación de dicha capacidad pueden diseñarse estructuras organizacionales óptimas, para lo cual COBIT, ITIL y TL9000 pueden servir como referencia, diseñar planes de capacitación, procedimientos y sistemas de gestión del conocimiento, establecer responsabilidades para la gestión de la información organizacional y divulgar las políticas TI, a través de un consistente sistema de comunicación, finalmente, es una buena práctica establecer variables de control. Para poder realizar la evaluación de capacidad es imprescindible que exista una estadística previa del rendimiento de la infraestructura TI y que se realice una comparación de dicha estadística con los indicadores de desempeño de las TI para el sector de la industria al que pertenece la organización. Un elemento a considerar, previo a la evaluación de capacidad, es el número de elementos de configuración alineados con los niveles de servicio respecto a: desempeño, seguridad y disponibilidad [16, 21-20].

Una vez definidas las políticas, se negociará con los responsables de los procesos de la organización los Acuerdos de Nivel de Servicio, según la secuencia de pasos que aparece en la Figura 3.
Todo cambio en la configuración en la infraestructura $\mathrm{TI}$, independientemente del nivel de complejidad que este tenga, debe estar gobernado por un conjunto de reglas de negocio que establecen quién chequea, bien sea desde el punto de vista del negocio o desde el punto de vista técnico, los cambios para su corrección; quién debe aprobarlos, quién debe implementarlos y cómo verificar que se han realizado correctamente o qué hacer si se descubre un problema. Es recomendable tener representadas estas relaciones en la base de datos de gestión de configuración (CMDB por las siglas en inglés de Configuration Management Data Base).

Esta fase tiene como objetivo la implementación de una arquitectura que facilite la construcción, evaluación y despliegue de políticas automatizables.

Para ello, tal y como se muestra en la Figura 6, la primera tarea consistirá en construir, desplegar y evaluar reglas operacionales. En este sentido, es necesario considerar que la condición establecida, puede contener un conjunto de cláusulas que darán como resultado una condición simple a partir de la cual puede evaluarse si se satisface o no. En cuanto a la cláusula de acciones, para el caso que esté previsto que se ejecuten varias acciones, se les establecerá la prioridad en función de la estrategia de ejecución. Así, las políticas se ordenan jerárquicamente definiendo

Tabla 2. Medidas relacionadas con las tareas en fase Planificación, Organización de Política.

\begin{tabular}{|l|l|}
\hline \multicolumn{2}{|c|}{ Planificación, Organización de Políticas } \\
\hline \multicolumn{1}{|c|}{ Tareas } & \multicolumn{1}{c|}{ Medidas Relacionadas } \\
\hline $\begin{array}{l}\text { Determinar las necesidades asociadas a } \\
\text { los catalizadores de las infraestructuras TI }\end{array}$ & $\begin{array}{l}\text { Necesidades asociadas a aplicaciones y servicios. } \\
\text { Necesidades asociadas a la automatización de procedimientos. } \\
\text { Necesidades asociadas a la gestión de la información. }\end{array}$ \\
& Necesidades de formación y completamiento del personal.
\end{tabular}

Fase: Ejecución de políticas. 


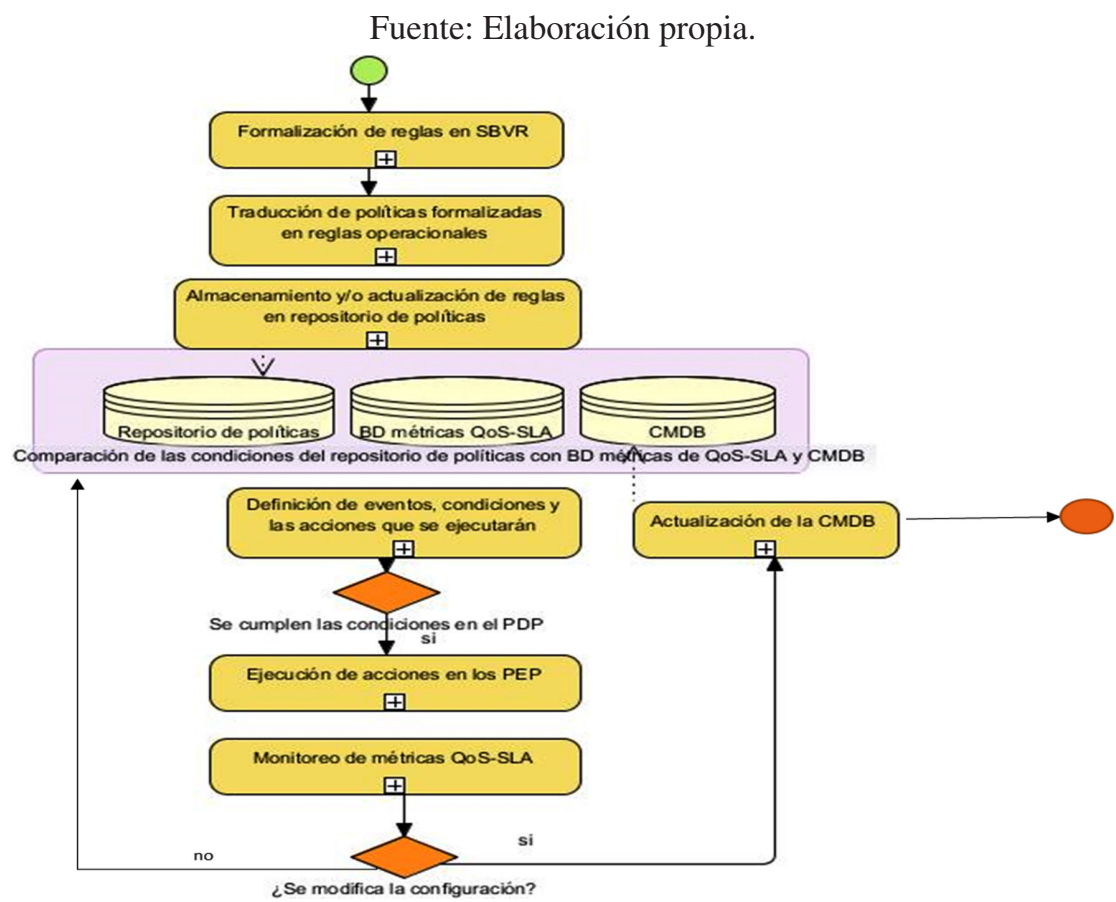

Figura 6. Ejecución de políticas.

reglas y subreglas. Además, deben existir eventos que desencadenen la evaluación de condiciones en una regla de políticas.

Al realizar la traducción de políticas automatizables a operaciones o comandos para cada tecnología implementada, es necesario consultar la base de datos de gestión de configuración, donde se registran los atributos de cada elemento de configuración (CI, por las siglas en inglés de Configuration Instance) durante su ciclo de vida, las relaciones que poseen con otros elementos y los registros vinculados a cada uno, por ejemplo, registros de incidentes, problemas o cambios [16]. Siendo CI cualquier elemento que se encuentra en la organización y que es importante controlar para proporcionar un servicio, estos van desde los servicios que ofrece el departamento de TI, hasta los elementos de hardware necesarios para su funcionamiento (máquinas, servidores, routers, periféricos, etc.), las personas involucradas, redes, software, documentación, proveedores, etc. De ahí que se instancian las reglas, primero para los servicios, luego para las plataformas y software que los soportan y, por último, para los elementos de hardware que aseguran esos servicios.
En el despliegue de la arquitectura PBNM, para la ejecución de políticas en la infraestructura, según recomienda el IETF, debe montarse una Aplicación de Gestión basada en Políticas que no es más que un sistema de administración Web que permite el monitoreo y control de los elementos que forman parte de la infraestructura que ejecuta las políticas. Dicha aplicación debe permitir al administrador, modelar las políticas que regirán el sistema haciendo uso del lenguaje de reglas de negocio de alto nivel SBVR (de las siglas en inglés de Semantics of Business Vocabulary and Business Rules). Estas reglas deben almacenarse en un repositorio de políticas, el cual constituye un almacén donde se registra y almacenan todas las políticas a ejecutar. Para ejecutar las acciones de gestión, se integra el módulo conocido como Punto de Decisión de Políticas (PDP) que, como su nombre indica, se especializa en tomar las decisiones de cuáles políticas se deben ejecutar y de su procesamiento, alineando de esta forma las necesidades del negocio al comportamiento coherente de la infraestructura y los servicios que corren sobre ella. Para este fin, el PDP transforma las políticas o reglas en representaciones operacionales aptas para ser interpretadas por los agentes de gestión que operan en los elementos de red. Este módulo debe 
contar con un motor de inferencia y se basa, para la toma de decisiones, en la información contenida en la base de datos de gestión de configuración y en las bases de datos donde se almacenan las métricas de desempeño de la infraestructura TI.

Para el despliegue en los recursos gestionados de las políticas o reglas, se recomienda utilizar las facilidades que ofrecen los agentes de gestión existentes, por ejemplo, el agente SNMP, sobre los cuales se deben implementar acciones de control, de esta manera se realiza un uso más eficiente de la red, por cuanto el monitoreo y el control se ejecutan desde un único agente.

Uno de los problemas a resolver utilizando PBNM es la gestión de la calidad de los servicios ya que las redes son sistemas complejos que soportan diferentes servicios, cada uno de los cuales, tienen requerimientos diferentes. Las soluciones PBNM basan su funcionamiento en reaccionar a determinadas condiciones con determinada acción. Sin embargo, alguna entidad centralizada en la organización debe determinar a cuáles recursos debe dárseles determinada prioridad sobre otros. Para esto, se propone modificar la arquitectura propuesta por el IETF, (ver Figura 7), creando una entidad capaz de evaluar el impacto de las políticas en los servicios, detectar conflictos y tomar acciones en caso de que estos ocurran, lo que permitirá priorizar unos servicios sobre el resto.

Esta nueva arquitectura permitirá evaluar a un nivel jerárquico superior el impacto de las políticas que

Tabla 3. Medidas relacionadas con las tareas en fase Ejecución de políticas.

\begin{tabular}{|l|l|}
\hline \multicolumn{2}{|c|}{ Ejecución de políticas } \\
\hline \multicolumn{1}{|c|}{ Tareas } & \multicolumn{1}{c|}{ Medidas Relacionadas } \\
\hline Construir, desplegar y evaluar reglas operacionales. & $\%$ de políticas convertidas en reglas operacionales. \\
\hline $\begin{array}{l}\text { Traducir políticas automatizables a operaciones o comandos } \\
\text { específicos para cada tecnología implementada. }\end{array}$ & $\begin{array}{l}\% \text { de políticas traducidas. } \\
\% \text { de recursos que aceptan la traducción. }\end{array}$ \\
\hline $\begin{array}{l}\text { Implementaraducidas que soporta cada recurso. } \\
\text { en la infraestructura }\end{array}$ & $\%$ de políticas automatizadas. \\
\hline
\end{tabular}

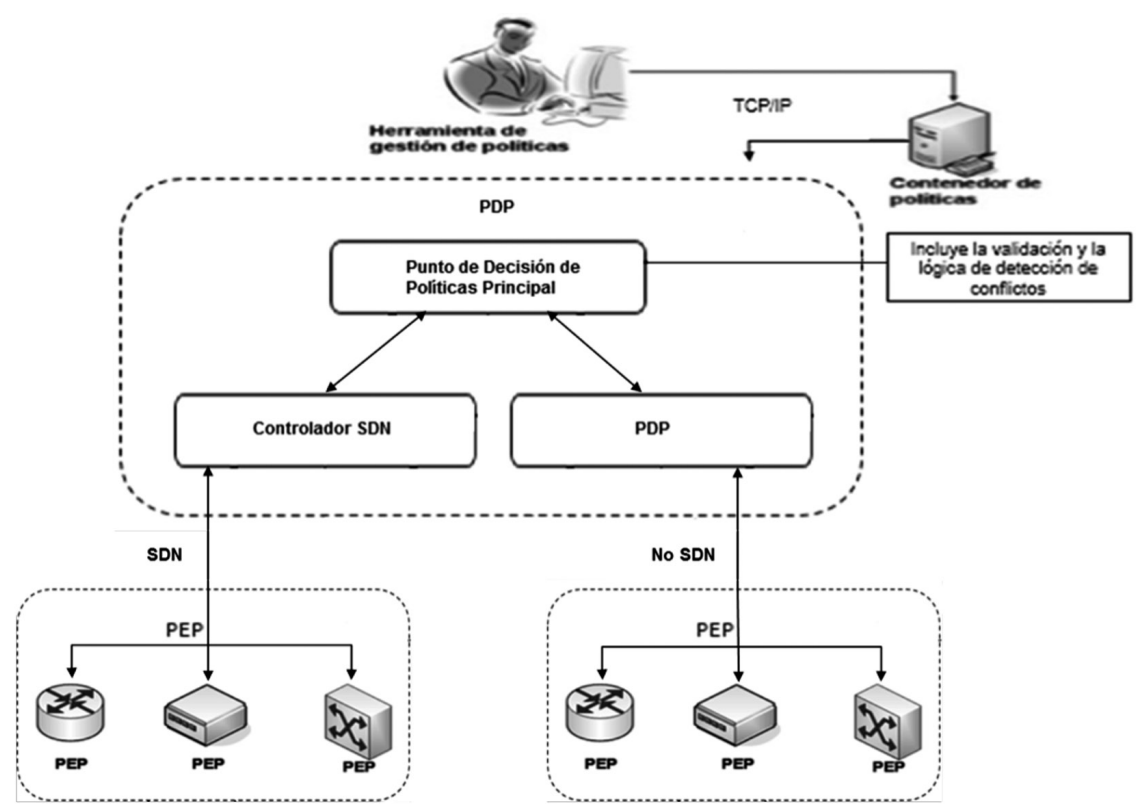

Figura 7. Arquitectura IETF modificada. 
se ejecutan, además en caso de definirse servicios críticos que afectan la continuidad del negocio, al afectarse, ejecutarían eventos que desencadenarían la evaluación de las políticas. En este caso, la entidad centralizada o Punto de Decisión de Políticas Principal evaluaría el comportamiento de los indicadores de desempeño globales de la infraestructura, y de existir alguna desviación, calcularía si tiene alguna política establecida para esa condición o emitiría alarmas al equipo de administración. Finalmente, calcularía la tasa de ocurrencia de los incidentes y en caso de exceder los valores predeterminados en los Acuerdos de Nivel de Servicio, notificaría al responsable del problema para desencadenar un proceso de evaluación de conflictos entre políticas.

La arquitectura modificada que se propone, realiza un monitoreo en tiempo real de las principales métricas de rendimiento utilizando un sistema de gestión que periódicamente consulta a los agentes desplegados en la infraestructura. Este verifica que el funcionamiento de las métricas previstas en los SLA sea correcto, y si existen violaciones de lo pactado, estas son tratadas y se generan reportes que son enviados al contacto establecido para el proceso afectado. Si este detecta que los servicios no están funcionando debidamente, tomará las medidas pertinentes y abrirá un reporte, lo procesará, dará solución y posteriormente, notificará la solución efectuada. Una media importante para evaluar el impacto de la ejecución de políticas sobre las infraestructuras TI, es medir el\% de nivel de servicio reportado de forma automatizada y el porciento de procesos relativos a las TI cubiertos por SLA.

Todas las fases se deben integrar a partir de la validación de una serie de medidas definidas. $\mathrm{Al}$ terminar la fase de planificación, organización y control, debe validarse que se cuenta con capacidades para proceder a la automatización de las políticas tal y como se muestra en la Tabla 2.
Es importante retroalimentar a la organización con un informe que contenga el cumplimiento de los SLA. De detectarse violaciones en el cumplimiento de los SLA, se procede a evaluar si están ocasionados por el proceso de ejecución de políticas, de planificación de políticas o si ocurrió durante el proceso de definición de políticas TI, en cuyo caso debe notificarse a la organización sobre las necesidades o políticas organizacionales que no pueden organizarse o establecerse a través de políticas TI para que se definan medidas organizativas.

\section{VALIDACIÓN}

Para demostrar la aplicabilidad del sistema, se realizó el estudio de un caso a partir de un experimento montado en un entorno simulado. En este se diseñaron un conjunto de políticas calidad de servicio a ejecutarse en el equipamiento activo desplegado sobre una arquitectura SND híbrida (ver Figura 8); tales políticas deben funcionar tanto para el segmento SDN híbrido como para el segmento conformado por equipamiento tradicional.

Dicha arquitectura se desplegó en un entorno de prueba simulado (ver Figura 9) empleando para ello el simulador GNS3 que permite lograr la emulación de dispositivos de redes, creando una plataforma que permite el fácil diseño de topologías de redes complejas [22-23]. Siendo consecuente con el procedimiento se evalúa la capacidad del entorno para configurar la arquitectura.

El escenario simulado representa una red LAN en la que se implementa un controlador que gestiona la red SDN a través del equipamiento híbrido identificado por el protocolo OpenFlow. Para simular el segmento de red SDN se utilizó el controlador SDN Floodlight debido, fundamentalmente, a que es un controlador multiplataforma muy popular, que brinda total soporte para el descubrimiento de los dispositivos (OpenFlow

Tabla 4. Medidas relacionadas con las tareas Evaluación del cumplimiento de SLA.

\begin{tabular}{|c|c|}
\hline \multicolumn{2}{|c|}{ Evaluación del cumplimiento de SLA } \\
\hline Tareas & \multicolumn{1}{|c|}{ Medidas Relacionadas } \\
\hline \multirow{2}{*}{ Evaluar el cumplimiento de SLA } & $\begin{array}{l}\% \text { de violaciones de niveles de servicio. } \\
\% \text { de nivel de servicio reportados de forma automatizada. } \\
\% \text { de procesos relativos a las TI cubiertos por SLA. }\end{array}$ \\
\hline
\end{tabular}


Fuente: Elaboración propia.

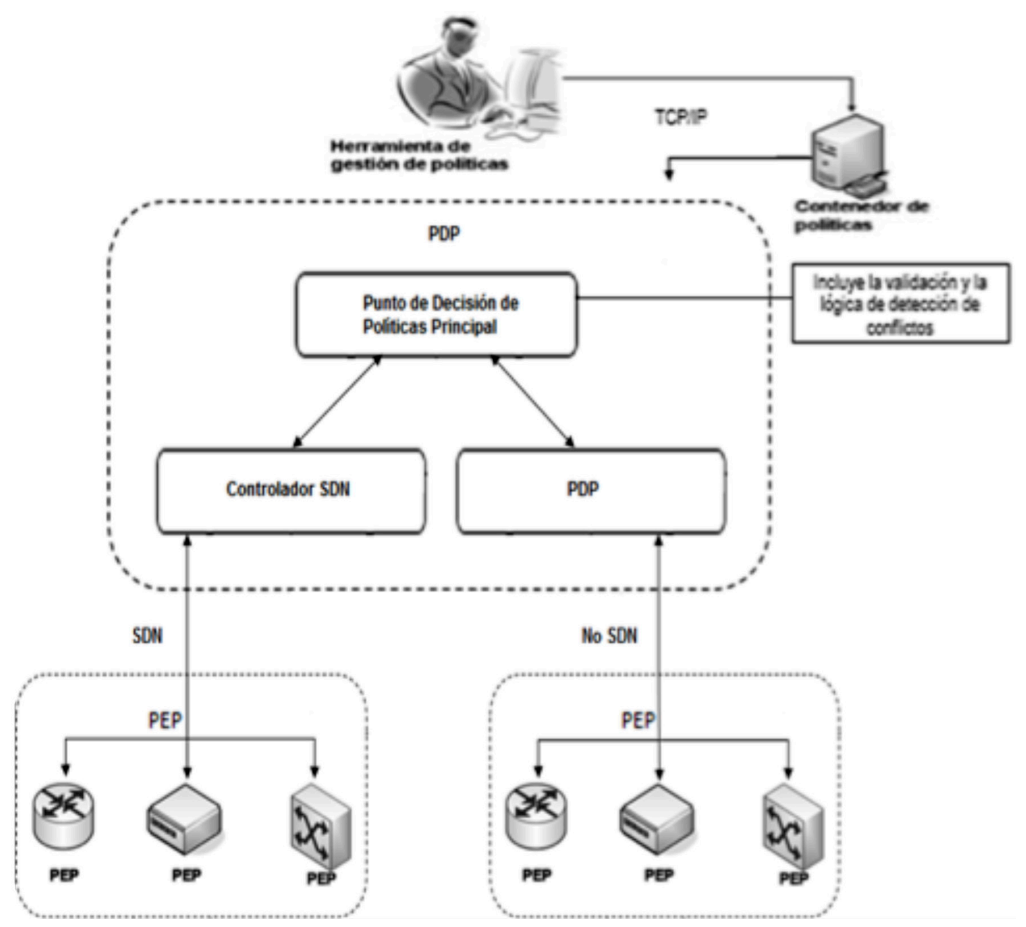

Figura 8. Arquitectura SDN-PBNM.

Fuente: Elaboración propia.

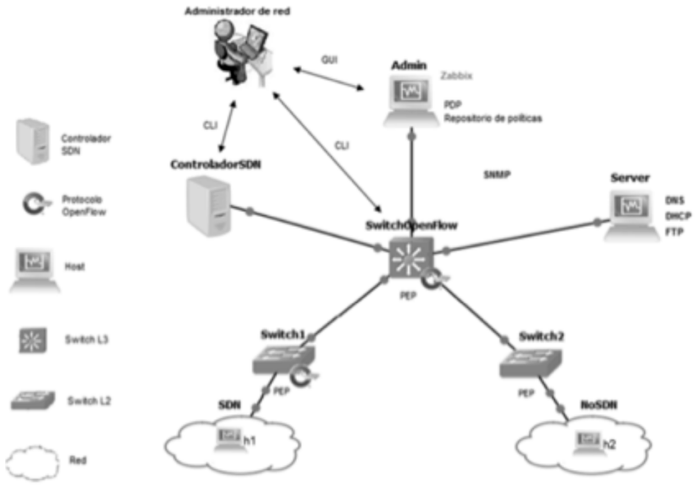

Figura 9. Escenario general de la arquitectura SDN-PBNM.

o no OpenFlow), gestión de los mismos, cálculo de rutas, acceso Web y otros. Además, incorpora una amplia lista de fabricantes de switches compatibles, incluye aplicaciones de ejemplo básicas como: hub, switch, flujo de red virtual, cortafuegos, entre otras, que permiten virtualizar varios dispositivos para la experimentación. En el escenario simulado, Floodlight se despliega en el host Controlador SDN a partir de una máquina virtual que posee, además del controlador, varias herramientas que permiten el monitoreo y el control de los recursos de red que este maneja. Entre estas herramientas están Mininet, Open vSwitch y Wireshark w/OpenFlow disector.

Se visualizan, además, un host denominado Admin sobre el que se implementan el repositorio de políticas y la aplicación de monitoreo y control de red Zabbix, y un host Server, el cual presta servicios como DNS, DHCP y FTP a la infraestructura de red tradicional. Tal y como se muestra en la Figura 10, el administrador de red realiza la configuración de los hosts a través de GUI (Graphic User Interface, por sus siglas en inglés) y del controlador y los switch de red mediante CLI (Command Line Interface, por sus siglas en inglés). Todos los dispositivos se interconectan utilizando enlaces Fast Ethernet. El PDP que compone la arquitectura SDN-PBNM va a ser implementados en el escenario de estudio a través de la herramienta Zabbix y las políticas que evalúan se almacenan en igualmente en dicha herramienta.

Zabbix define un conjunto de funcionalidades que permiten el monitoreo de la configuración de los 
dispositivos que tiene registrados en un inventario de activos de red disponibles. Estas funcionalidades denominadas Disparadores o Triggers identifican cambios de estado en los dispositivos, a partir de reglas de discriminación que permiten generar alertas. Estas reglas evalúan condiciones que el usuario define y que determinan el comportamiento que tomará el sistema. Conforme se generan las alertas, el sistema puede ejecutar acciones automáticamente en base a las restricciones que fueron predefinidas, que pueden ser enviar un mensaje o ejecutar un comando remoto a través de SSH (por las siglas en inglés de Secure Shell), Telnet (por las siglas en inglés de Telecommunication Network), IPMI (por las siglas en inglés de Intelligent Platform Management Interface) o mediante algún programa informático cuya función consista en interpretar órdenes conocido como bash (por las siglas en inglés de Bourne again shell). Estas acciones que se evalúan y ejecutan constituyen las políticas que precisan el comportamiento de la red en la arquitectura SDN-PBNM planteada.

Al realizar una solicitud ICMP (por las siglas en inglés de Internet Control Message Protocol) desde el nodo h1 a h2 mediante el comando ping de h1 a h2 se puede apreciar cómo se establece la comunicación entre los dispositivos especificados mediante el protocolo OpenFlow. Con esto se comprueba el correcto funcionamiento de Floodlight. Para probar la arquitectura, se apaga el controlador SDN y se verifica que no hay conexión entre los nodos h1 y h2

La Figura 10 representa una de las políticas que se implementan en el PDP para restaurar la configuración tradicional del equipamiento de red híbrido en caso de una falla en la disponibilidad del controlador SDN.

En este caso, para ejecutar la política de Restaurar configuración Switches después de una falla en el controlador se evalúan los disparadores siguientes:

A. Trigger value $=$ PROBLEM $($ El estado del disparador es un problema).

B. Trigger $<>$ Server: FTP Service is down on server (El servicio FTP alojado en el host Server está caído).

C. Trigger $=$ Controlador SDN: Controlador SDN no se encuentra disponible.

Cuando la expresión lógica definida por (A and (not $\mathrm{B}$ and $\mathrm{C}$ )) es verdadera entonces se aplica la acción de ejecutar un script basado en bash almacenado en el host Admin que restaura la configuración de los dispositivos, la cual se encuentra almacenada en el servidor FTP del host Server.

En este escenario se ejecutan otras políticas específicas como parte de la evaluación de la propuesta de arquitectura para la gestión de SDN

Fuente. Elaboración propia.

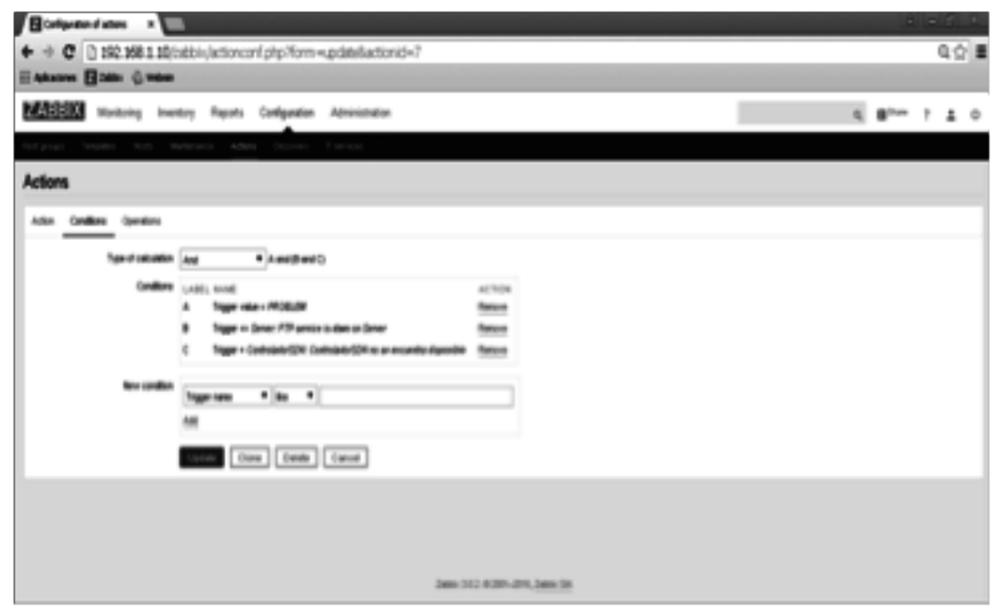

Figura 10. Política definida para resolver fallo de disponibilidad en el controlador SDN. 
híbrida. Las mismas se ejecutan a través de scripts basados en bash que realizan procedimientos remotos en los PEP. Estas políticas son:

- QoS: Evalúa valores de rendimiento obtenidos mediante los agentes SNMP en los PEP y aplica sobre los mismos, configuraciones de balanceo de carga.

- Salva centralizada: Realiza la operación de salva del estado funcional actual de los PEP en un servidor FTP.

\section{CONCLUSIONES}

La gestión de infraestructuras TI, se concentra en la aplicación de medidas de control tecnológico y no en la aplicación de políticas de la organización sobre dichas infraestructuras, lo que garantiza el alineamiento entre la operación de las infraestructuras de las Tecnologías de la Información y los objetivos de la organización.

El procedimiento para la ejecución de políticas de la organización sobre infraestructuras TI, contribuyó a garantizar el aseguramiento tecnológico de los procesos claves de una organización, permitiendo: realizar una gestión integrada basada en políticas, la traducción de las reglas a las características de cada uno de los equipos y la solución de conflictos entre reglas, lo que facilita el alineamiento a través de la gestión de los Acuerdos de Nivel de Servicio. Adicionalmente, permitió la mejora continua en los servicios.

El uso de la arquitectura PBNM modificada que se propone permitió la detección de conflictos entre políticas y evaluación del impacto de éstas sobre la infraestructura TI.

El empleo en una arquitectura SDN híbrida, permitió garantizar la continuidad de servicio ante la falla del controlador SDN, eliminando la existencia de un punto único de fallos en dicha arquitectura.

\section{REFERENCIAS}

[1] Z. Alreemy, V. Chang, R. Walters and G. Wills. "Critical success factors (CSFs) for information technology governance (ITG)". International Journal of Information Management. Vol. 36 Nº 6 Part A, pp. 907-
916. Diciembre 2016. ISSN: 0268-4012. DOI: 10.1016/j.ijinfomgt.2016.05.017.

[2] G. Krishnan and V. Ravindran. "IT service management automation and its impact to IT industry”. 2017. International Conference on Computational Intelligence in Data Science (ICCIDS). pp. 1-4. 2017. DOI: 10.1109/ ICCIDS.2017.8272633

[3] A.C. Riekstin, G.C. Januário, B.B. Rodrigues, V.T. Nascimento, T.C.M. DB. Carvalho and C. Meirosu. "A Survey of Policy Refinement Methods as a Support for Sustainable Networks". IEEE Communications Surveys Tutorials. Vol. $18 \mathrm{~N}^{\circ}$ 1, pp. 222-235. Firstquarter 2016. ISSN: 1553-877X. DOI: 10.1109/COMST.2015.2463811.

[4] Y. Kryftis, M. Grammatikou, D. Kalogeras, and V. Maglaris. "Policy-Based Management for Federation of Virtualized Infrastructures". Journal of Network and Systems Management. Vol. $25 \mathrm{~N}^{\circ}$ 2, pp. 229-252. Abril 2017. ISSN: 1064-7570, 1573-7705. DOI: $10.1007 /$ s10922-016-9390-z.

[5] J. Lourenço, C. Santos-Pereira, R. Rijo and R. Cruz-Correia. "Service Level Agreement of Information and Communication Technologies in Portuguese Hospitals". CENTERIS 2014 - Conference on ENTERprise Information Systems / ProjMAN 2014 - International Conference on Project MANagement / HCIST 2014 - International Conference on Health and Social Care Information Systems and Technologies. Vol. 16, pp. 1397-1402, 2014. ISSN: 2212-0173. DOI: 10.1016/j. protcy.2014.10.158.

[6] N. Sfondrini, G. Motta and L. You. "Service level agreement (SLA) in Public Cloud environments: A Survey on the current enterprises adoption". 2015 5th International Conference on Information Science and Technology (ICIST). pp. 181-185. 2015. DOI: 10.1109/ICIST.2015.7288964.

[7] J.S. Strassner, S. Van der Meer, Donnelly, and M. Ó. Foghlú. "The Design of a New Context-Aware Policy Model for Autonomic Networking". International Conference on Autonomic Computing. Chicago. pp. 119-128. 2008. DOI: 10.1109/ ICAC.2008.36.

[8] S. Davy, B. Jennings and J. Strassner. "The policy continuum-Policy authoring and 
conflict analysis". Computer Communications. Vol. $31 \mathrm{~N}^{\circ} 13$, pp. 2981-2995. Agosto 2008. ISSN: 0140-3664. DOI: $10.1016 / \mathrm{j}$. comcom.2008.04.018

[9] J. Strassner. "Context-aware dynamic policy selection for load balancing behavior". Google Patents. 28 marzo de 2014

[10] J. Strassner. "DEN-ng: achieving businessdriven network management". Management Solutions for the New Communications World. IEEE/IFIP. pp. 753-766. Florence, Italy. 2002. ISBN: 0-7803-7382-0. DOI: 10.1109/NOMS.2002.1015535.

[11] C.C. Machado, J.A. Wickboldt, L.Z. Granville and A. Schaeffer-Filho. "ARKHAM: An Advanced Refinement toolkit for Handling Service Level Agreements in SoftwareDefined Networking". Journal of Network and Computer Applications. Vol. 90, pp. 1-16. Julio 2017. ISSN: 1084-8045. DOI: 10.1016/j.jnca.2017.04.009.

[12] K. Odagiri, S. Shimizu, N. Ishii and M. Takizawa. "Load Experiment of the Cloud Type Virtual Policy Based Network Management Scheme for the Common Use between Plural Organizations". 2017 31st International Conference on Advanced Information Networking and Applications Workshops (WAINA). pp. 296-301. 2017. DOI: 10.1109/WAINA.2017.16.

[13] J. Strassner. "Policy-based network management: solutions for the next generation". CERN Document Server. 2003. [En línea]. Disponible en: http://cds.cern.ch/ record/1991797. [Accedido: 22-feb-2018].

[14] F.F. Moghaddam, P. Wieder and R. Yahyapourz. "Policy Engine as a Service (PEaaS): An Approach to a Reliable Policy Management Framework in Cloud Computing Environments". 2016 IEEE 4th International Conference on Future Internet of Things and Cloud (FiCloud). pp. 137144. 2016. DOI: 10.1109/FiCloud.2016.27
[15] M. Mohamed, O. Anya, S. Tata, N. Mandagere, N. Baracaldo and H. Ludwig. "rSLA: An Approach for Managing Service Level Agreements in Cloud Environments". International Journal of Cooperative Information Systems. Vol. $26 \mathrm{~N}^{\circ} 02$, pp. 1742003. 2017.ISSN: 0218-8430. DOI: 10.1142/S0218843017420035.

[16] P. Han, Y. Liu and L. Guo. "QoS Satisfaction Aware and Network Reconfiguration Enabled Resource Allocation for Virtual Network Embedding in Fiber-Wireless Access Network". Computer Networks. Junio 2018.ISSN: 13891286. DOI: 10.1016/j.comnet.2018.06.019

[17] R.F. Escobar y G.M.T. Bermúdez. "Implementación de procedimientos de gobernabilidad TI en la red de investigación de tecnología avanzada basado en ITIL, COBIT y la ISO 20000-27000". Redes de Ingeniería. Vol. 6. Septiembre 2015. ISSN: 2248-762X. DOI: $10.14483 / 2248762 X .8501$.

[18 P. Muni, S. Roy, Y.T.Y.J.J.L. Chiang, A.J.M. Viallet, y N. Budhiraja. "Recommending Resolutions of ITIL Services Tickets Using Deep Neural Network". en Proceedings of the Fourth ACM IKDD Conferences on Data Sciences. New York, USA. pp. 14:1-14:10. 2017. ISBN: 978-1-4503-4846-1. DOI: 10.1145/3041823.3041831.

[19] D.N.L. Armendáriz. "Modelo de gestión de los servicios de tecnología de información basado en COBIT, ITIL e ISO/IEC 27000". Rev. Tecnológica - ESPOL. Vol. $30 \mathrm{~N}^{\circ} 1$. Mayo 2017. ISSN: 1390-3659.

[20] S. Cocanguilla y P. Santino. "Diseño e implementación de un modelo para el control de las rutinas de operación y mantenimiento de acuerdo a las mejoras prácticas ETOM, ITIL Y COBIT para la red IP/MPLS de la Corporación Nacional de Telecomunicaciones". Universidad de las Fuerzas Armadas ESPE. Carrera de Ingeniería Electrónica en Redes y Comunicación de Datos. 2016. 